\title{
Statistical physics applied to stone-age civilization
}

September 26, 2021

\author{
M.A. Sumour ${ }^{1}$, M.A. Radwan ${ }^{1}$, M.M. Shabat ${ }^{2}$, Ali H. El-Astal ${ }^{1}$ \\ ${ }^{1}$ Physics Department, Al-Aqsa University, P.O.4051, Gaza, Gaza Strip, Pales- \\ tinian Authority, e-mail: msumoor@yahoo.com , ma.radwan@alaqsa.edu.ps , \\ a_elastal@yahoo.com . \\ 2 Physics Department, Islamic University, P.O.108, Gaza, Gaza Strip, Pales- \\ tinian Authority, e-mail : shabat@iugaza.edu.ps
}

\begin{abstract}
About 45,000 years ago, symbolic and technological complexity of human artefacts increased drastically. Computer simulations of Powell, Shennan and Thomas (2009) explained it through an increase of the population density, facilitating the spread of information about useful innovations. We simplify this demographic model and make it more similar to standard physics models. For this purpose, we assume that bands (extended families) of stone-age humans were distributed randomly on a square lattice such that each lattice site is randomly occupied with probability $p$ and empty with probability $1-p$. Information spreads randomly from an occupied site to one of its occupied neighbours.

If we wait long enough, information spreads from one side of the lattice to the opposite site if and only if $p$ is larger than the percolation threshold; this process was called "ant in the labyrinth" by de Gennes 1976. We modify it by giving the diffusing information a finite lifetime, which shifts the threshold upwards
\end{abstract}

\section{Introduction:}

Modern humans presumably originated about 200,000 years ago in East Africa. They emigrated to $150 \mathrm{~km}$ north of Gaza about 100,000 years ago (Skhul near Haifa) but died out there again. Then about 50,000 years ago they again emigrated from Africa, this time more successfully, but presumably again via Gaza and Haifa.

According to [1], demographic factors can explain geographic variation in the timing of the first appearance of modern behaviour without invoking increased cognitive capacity. 
Now we try to reproduce this demographic effect by applying standard percolation theory [2]. Only for densities p above the percolation threshold $\left(p_{c}=0.593\right.$ on infinite square lattices) can information travel from one side of the lattice, via random jumps to occupied nearest-neighbour sites, up to the opposite side of the lattice. This type of random walk on random square lattices was discussed a lot about three decades ago, but mostly for large systems and long times close to the percolation threshold. We will show that for times below 10,000 jump attempts even for quite small lattices, one needs occupation probabilities far above the percolation threshold $\left(p_{c}=0.593\right)$ in order to transfer information from one side of the lattice to the opposite site.

\section{Data and Simulations:}

First we have occupy a square lattice of size $L x * L y$ randomly with probabilities $p$ for occupied and $1-p$ for empty, clusters are groups of occupied neighbouring sites. Then we put $N$ diffusing particles randomly onto occupied sites. Then we let each particle at each time step try to move into one of the four possible directions; if that neighbour is occupied the particle (which may represent a teacher of new techniques) moves there, if it is empty the particle stays at its old place.

In this way, $N$ particles diffuse on a randomly occupied lattice, using only the occupied lattice sites. The occupied sites can be settlements of humans, and the diffusing particles can be visitors spreading information on new techniques. We start our simulations with for different $L x=10,20,30,40,50,100,200,300$. With $L y=100,1000$, with different probability $=0.60,0.70,0.80,0.90$. We check after which time, i.e. after how many jump attempts, the particle diffuses across the lattice in $\mathrm{x}$-direction.

And we have nine samples $(N=9)$ ( $N$ diffusing particles on the same lattice), from which the median is defined such that four times are larger and four times are smaller than the median time, shown in Fig. 1. 


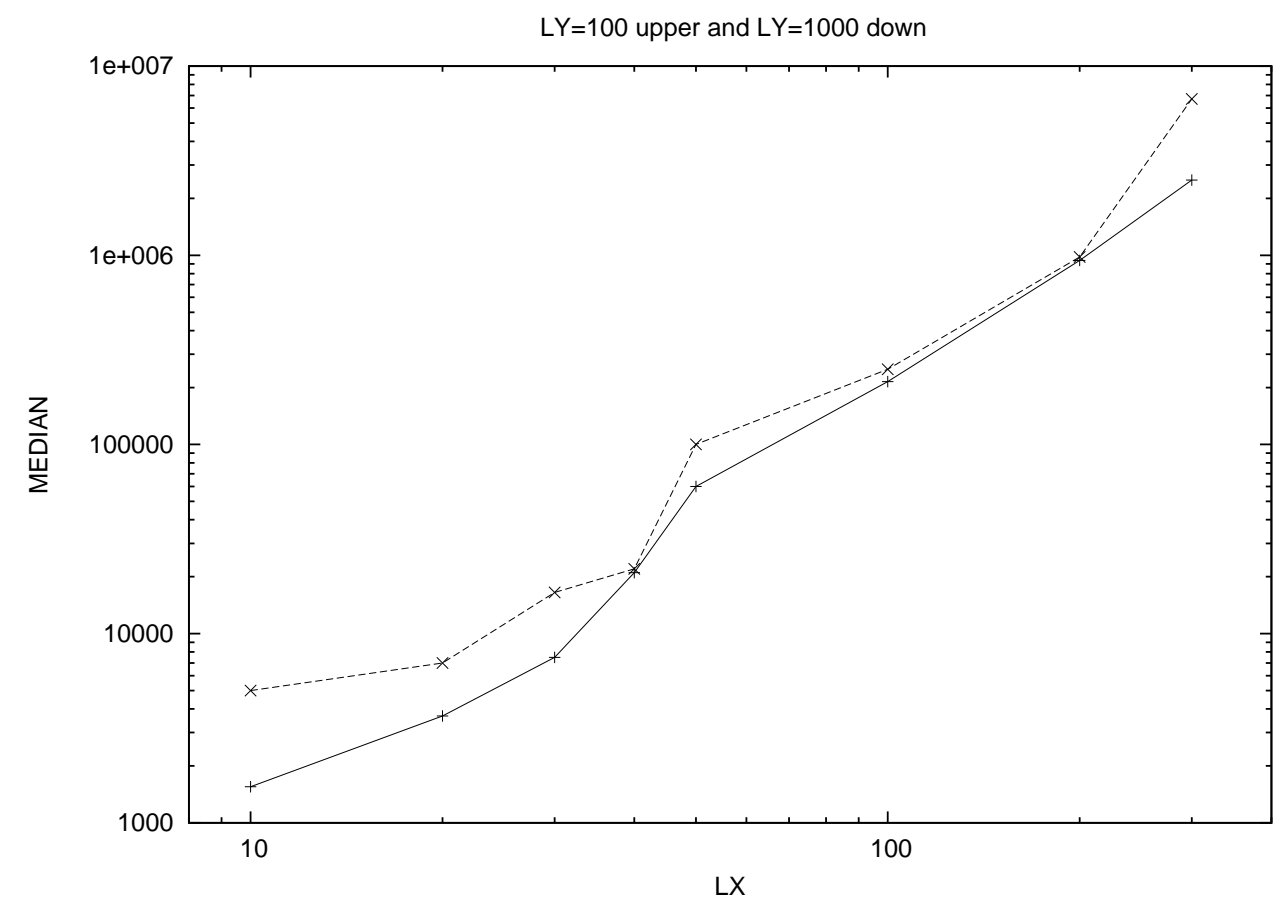

Figure 1: Median time versus $L x$ for $L y=100$ upper line and the down line for $L y=1000$, iseed $=1$, at $p=0.64$.

Then we complete our simulations for three probabilities $=0.70,0.80,0.90$ and take the median time, $L y=100$ with random seed number iseed=1, so we get figure (2): 


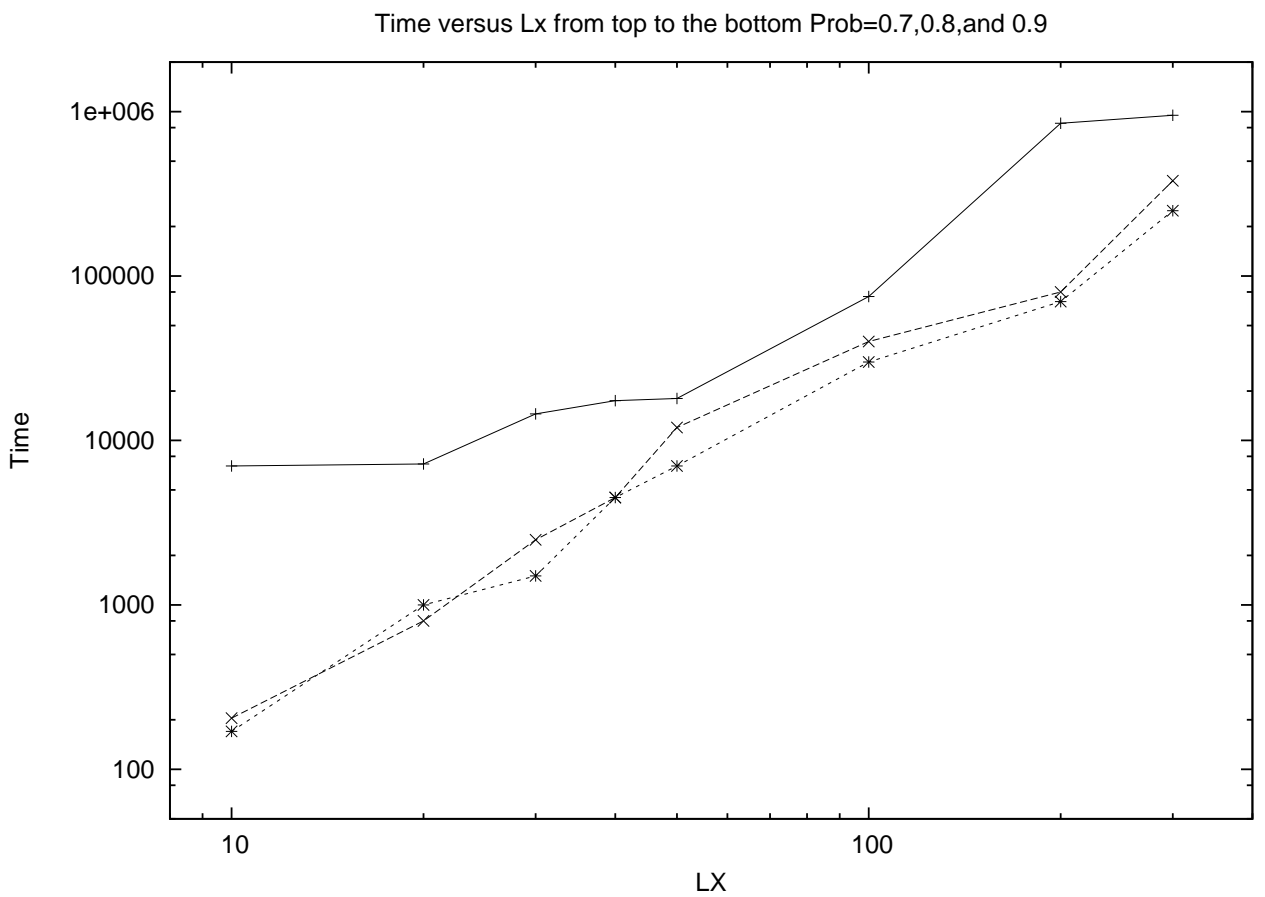

Figure 2: Median time versus $L x$ for $L y=100$ from top to bottom prob. $=$ $0.70,0.80, \& 0.90$ with iseed $=1$.

Figure (3) change the random number as iseed $=1,2,3$ for one sample of probability $=0.70$, and then we take the average median for each iseed number to get figure (3): 


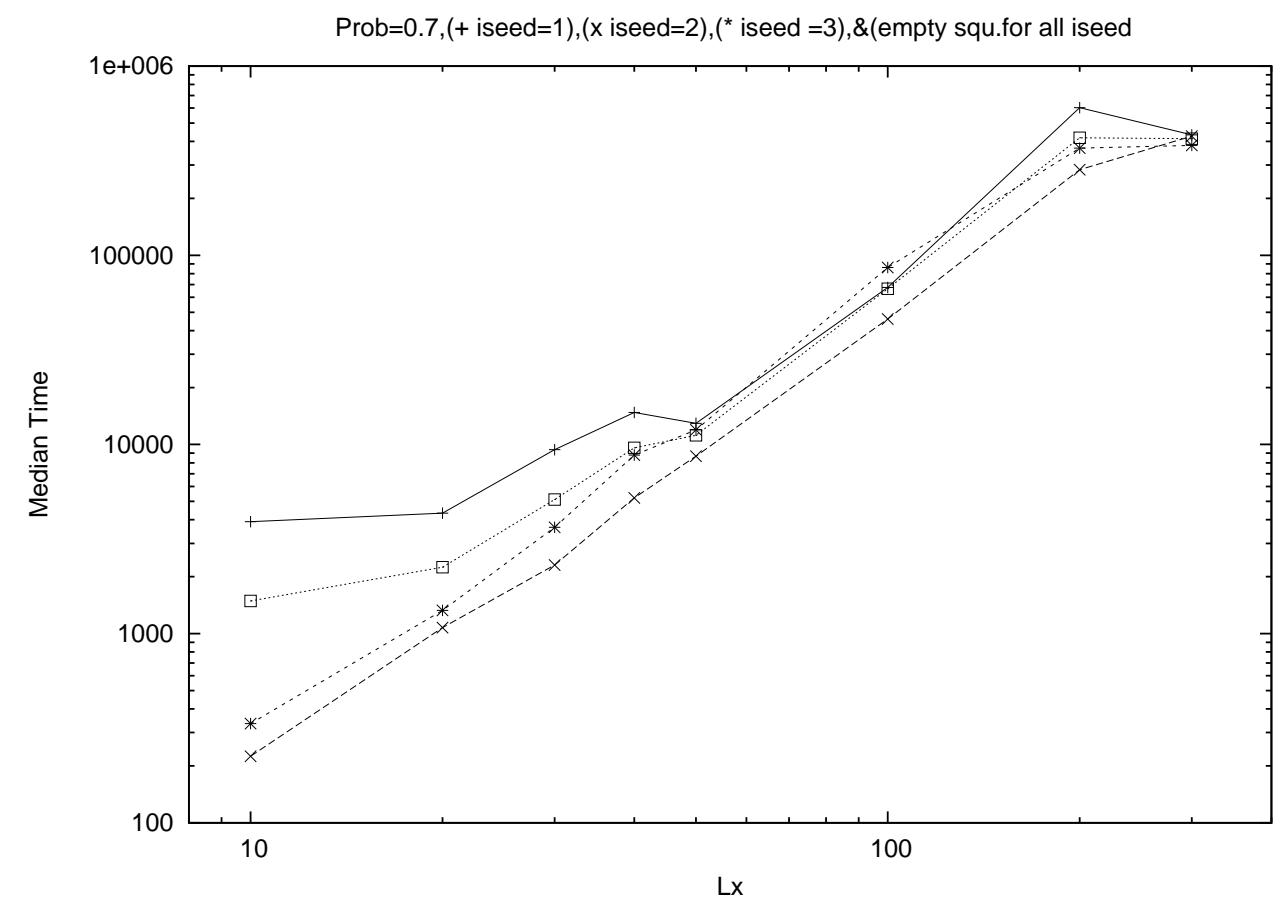

Figure 3: Median time for $p=0.7$ fluctuations are reduced by averaging over three random lattices for $L y=100$.

\section{Conclusions:}

If we identify one time unit with one day, times above 10,000 are unrealistic for a single messenger of new techniques. Thus only rather large occupation probabilities closer to unity than to $p_{c}$ allow the random spread over dozens of distances between human bands. Future simulations might facilitate information spread by allowing these bands to move randomly on the lattice (annealed instead of quenched disorder), as was appropriate before the Neolithicum with agriculture.

\section{Acknowledgements:}

The authors would like to thank Prof. Stauffer for many valuable suggestions, fruitful discussions and constructive advice during the development of this work.

\section{References:}

[1] Adam Powell, Stephen Shennan, and Mark G. Thomas, Science 324, 1298 (2009). 
[2]Dietrich Stauffer and Amnon Aharony, Introduction to Percolation Theory, Taylor and Francis, London 1994 (2nd printing of 2nd edition) 